\title{
Development of a PD-L1-Expressing Orthotopic Liver Cancer Model: Implications for Immunotherapy for Hepatocellular Carcinoma
}

\author{
Da-Liang Ou ${ }^{a} \quad$ Yu-Yang Lin ${ }^{b} \quad$ Chia-Lang Hsu ${ }^{c} \quad$ Yin-Yao Lin ${ }^{a}$ \\ Chia-Wei Chen $^{a}$ Jhang-Sian $\mathrm{Yu}^{d}$ Shi-Chuen Miaw ${ }^{d}$ Ping-Ning Hsu ${ }^{d}$ \\ Ann-Lii Cheng ${ }^{\mathrm{a}, \mathrm{e}-\mathrm{g}}$ Chiun Hsu ${ }^{\mathrm{a}}$ e-g \\ a Graduate Institute of Oncology, National Taiwan University College of Medicine, Taipei,

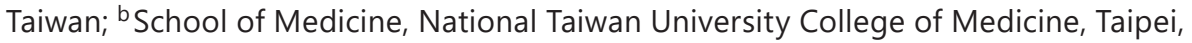 \\ Taiwan; ' Department of Medical Research, National Taiwan University Hospital, Taipei, \\ Taiwan; ${ }^{\mathrm{d}}$ Graduate Institute of Immunology, National Taiwan University College of Medicine, \\ Taipei, Taiwan; ${ }^{e}$ National Taiwan University Cancer Center, Taipei, Taiwan; ${ }^{\mathrm{f}}$ Department of \\ Oncology, National Taiwan University Hospital, Taipei, Taiwan; ${ }^{9}$ Department of Internal \\ Medicine, National Taiwan University Hospital, Taipei, Taiwan
}

\section{Keywords}

Anti-programmed cell death-1 - Sorafenib · CD8+ T cells · Tumor-infiltrating lymphocytes . Immune checkpoint inhibitor

\begin{abstract}
Background: Anti-programmed cell death-1(anti-PD1) treatment has shown promising antitumor efficacy in patients with advanced hepatocellular carcinoma (HCC). This study sought to explore the functional significance of programmed death ligand-1 (PD-L1) expression in tumor cells in the tumor microenvironment. Methods: The mouse liver cancer cell line BNLMEA was transfected with PD-L1 plasmids and stable clones expressing PD-L1 were selected. An orthotopic HCC model was generated by implanting the cells into the subcapsular space of BALB/c mice. Cell growth features were measured by proliferation assay, colony formation,
\end{abstract}

Dr. Da-Liang Ou is a National Taiwan University YongLin Scholar since 2018. Part of this study has been presented at the third CRI-CIMT-EATI-AACR Cancer Immunotherapy Conference, September 6-9, 2017, in Frankfurt, Germany. 


\section{Liver Cancer}

flow cytometry (in vitro), ultrasonography, and animal survival (in vivo). The changes in T-cell function were examined by cytokine assay, expression of T-cell related genes, and flow cytometry. The efficacy of anti-PD1 therapy was compared between the parental and PD-L1expressing tumors. Results: PD-L1 expression did not affect growth characteristics of BNLMEA cells but downregulated the expression of genes related to T-cell activation in the tumor microenvironment. Co-culture of PD-L1-expressing BNL-MEA cells with CD8+ T cells reduced T-cell proliferation and expression of cytokines IFN $\gamma$ and TNF $\alpha$. Tumors with PD-L1 expression showed better response to anti-PD1 therapy and depletion of CD8+ T cells abolished the antitumor effect. The difference in treatment response between parental and PD-L1-expressing tumors disappeared when a combination of anti-PD1 and sorafenib was given. Conclusions: PD-L1 expression in HCC cells may inhibit T-cell function in the liver tumor microenvironment. Anti-PD1 therapy appeared more effective in PD-L1-expressing than nonexpressing tumors, but the difference was diminished by the addition of sorafenib.

(c) 2018 S. Karger AG, Basel

\section{Introduction}

Immuno-oncology is a new area of drug development that focuses on enhancing the host's effective anticancer immune response. A major breakthrough has been achieved in agents targeting specific checkpoint proteins that play critical roles in regulating T-cell activation and proliferation [1]. The anti-programmed cell death-1 (anti-PD1)/anti-programmed death ligand-1 (anti-PD-L1) antibodies have shown promising antitumor activity and have been approved for the treatment of many cancer types [2]. Current research focuses on the development of combination treatment for better treatment efficacy and predictive biomarkers for patient enrichment [3].

Hepatocellular carcinoma (HCC) is still a major cancer killer worldwide, and options of systemic therapy for advanced HCC are limited [4, 5]. HCC is characterized by its association with chronic inflammation and an immune-suppressive tumor microenvironment, which makes immunotherapy an attractive approach for new drug development [6]. Results from early-phase clinical trials of anti-PD1 and anti-CTLA4 treatment have demonstrated promising antitumor activity and the anti-PD1 agent nivolumab recently obtained accelerated approval from the US Food and Drug Administration for the treatment of advanced HCC patients who had experienced sorafenib treatment [7-9]. The development of immune checkpoint inhibitor therapy, particularly anti-PD1/anti-PD-L1 therapy, and other immunomodulatory approaches are likely to change the landscape of drug development for HCC.

PD-L1 expression is the most extensively studied predictive biomarker for the efficacy of anti-PD1/anti-PD-L1 treatment [10-12]. High PD-L1 expression has been demonstrated to be associated with better response and improved survival in patients with melanoma, nonsmall cell lung cancer, and head and neck squamous cell carcinoma after anti-PD1 therapy [13-15]. However, there are still many unresolved issues regarding the interpretation of PD-L1 expression status, such as the cutoff value to define positivity, the relevance of PD-L1 expression on cancer versus immune cells, and the temporal and spatial heterogeneity of PD-L1 expression [16, 17]. PD-L1 expression in tumor cells occurred in about $20 \%$ of HCC patients [18], but its association with response to anti-PD1 therapy was not clear [8]. Therefore, improved understanding of the role of PD-L1 expression in the HCC tumor microenvironment may help identify patients who will benefit more from anti-PD1 therapies and design future combination strategies. 


\section{Liver Cancer}

\begin{tabular}{l|l}
\hline Liver Cancer 2019;8:155-171 \\
\hline DOI: 10.1159/000489318 & $\begin{array}{l}\text { @) 2018 S. Karger AG, Basel } \\
\text { www.karger.com/lic }\end{array}$ \\
\hline
\end{tabular}

Ou et al.: PD-L1 Expression in Liver Cancer

PD-L1 expression in cancer cells may be constitutive, i.e., regulated by genetic events in cancer cells, or inductive, i.e., stimulated by immune response in the tumor microenvironment [11]. Animal models with constitutive PD-L1 expression have been generated to study the roles of PD1/PD-L1 signaling in immune regulation in the tumor microenvironment [19-23]. In the present study, we establish a mouse model with orthotopic liver tumors that express PD-L1 to explore the impact of PD-L1 expression on growth characteristics, regulation of the immune microenvironment, and potential association with the efficacy of anti-PD1 therapy in HCC.

\section{Materials and Methods}

Establishment of PD-L1-Expressing Mouse Liver Cancer Cells

The mouse liver cancer cell lines BNL-MEA and Hepa1-6, obtained from the American Type Culture Collection (ATCC), were cultured in Dulbecco's modified Eagle's medium (DMEM) supplemented with 10\% fetal bovine serum, penicillin (100 units $/ \mathrm{mL})$, and streptomycin $(100 \mu \mathrm{g} / \mathrm{mL})$. The cells were maintained in a humidified incubator under $5 \% \mathrm{CO}_{2}$ at $37^{\circ} \mathrm{C}$. PD-L1 expression was measured by western blotting and immunofluorescence assay (detailed information about the antibodies used is listed in the supplementary material; for all online suppl. material, see www.karger.com/doi/10.1159/000489318). A panel of human HCC cell lines was used for comparison (see online supplementary material). Additional information on the HCC cell lines culture, western blot analysis, and immunofluorescence staining can be found in the online supplementary Materials and Methods.

The BNL-MEA cell line was selected for establishing PD-L1-expressing clones because of low endogenous PD-L1 expression. PD-L1 or empty plasmids (MR203953 and PS100001; Origene Technologies, Rockville, MD, USA) were transfected into the BNL-MEA cells using a PolyJet transfection reagent (Signagen Laboratories, Rockville, MD, USA) and stable clones were selected by G418 treatment (Life Technologies). PD-L1 expression was confirmed by western blotting and immunofluorescence assay.

In vitro growth characteristics of PD-L1-expressing and parental BNL-MEA cells were compared by cell viability (MTT [3-(4,5-dimethylthiazol-2-yl)-2,5-diphenyltetrazolium] assay), cell proliferation (3-dimensional [3D] culture), apoptosis and cell cycle (flow cytometry), and colony formation assay. For the 3D proliferation assay, the cells were seeded into the Perfecta3 $\mathrm{D}^{\circledR}$ hanging drop plate (3D Biomatrix, Ann Arbor, MI, USA) at a density of 1,000 cells/well and allowed to grow for 3 and 7 days to form 3D spheroids. Optical microscopic images of 3D spheroids were taken using an inverted fluorescence microscope with a Nikon Eclipse TS100 camera. Spheroids were harvested into the standard 96-well plates, and measured by MTT assay. The cell cycle distribution and extent of apoptosis were evaluated by staining the cells with propidium iodide ( $50 \mu \mathrm{g} / \mathrm{mL})$ in the presence of RNase A (100 units $/ \mathrm{mL}$ ) and measured by flow cytometry [24]. For the colony formation assay, the cells were seeded into 6 -well plates $\left(6 \times 10^{3}\right.$ cells/well $)$ and maintained in medium for 14 days, with medium changes every 4 days. Colonies were fixed with methanol and stained with $0.1 \%$ crystal violet (Sigma-Aldrich, St. Louis, MO, USA) in PBS for $15 \mathrm{~min}$. The number of stained colonies per well was quantified by ImageJ software in triplicate for each treatment group.

\section{Exploration of Immune Modulatory Effects of PD-L1 Expression by Liver Cancer Cells}

To evaluate the effects of PD-L1 expression by liver cancer cells on cytotoxic T-cell function, CD8+ T cells were purified from spleens and lymph nodes of 6- to 8-week-old male BALB/c mice using the EasySep Mouse CD4 and CD8a Positive Selection Kit (StemCell Technologies, Vancouver, BC, Canada) and enriched as previously described [25]. The enriched CD4+ and CD8+ T cells were first rested in complete RPMI 1640 medium for $24 \mathrm{~h}$, then co-cultured with parental and PD-L1-expressing BNL-MEA cells in different ratios for cytokine and cell proliferation assays.

For the cytokine assay, the CD4+ and CD8+ T cells, co-cultured with parental and PD-L1-expressing BNL-MEA cells, were stimulated with Dynabeads ${ }^{\circledR}$ CD3/CD28 magnetic beads (Gibco) for $24 \mathrm{~h}$. The culture supernatants were then collected, and the concentrations of IFN $\gamma$ and TNF $\alpha$ were quantified by ELISA (eBioscience). For the T-cell proliferation assay, CD4+ and CD8+ T cells were first labeled with $5 \mu \mathrm{M}$ carboxyfluorescein succinimidyl ester (CFSE) (Invitrogen) in prewarmed PBS for $3 \mathrm{~min}$ at $37^{\circ} \mathrm{C}$. The CFSE-labeled CD4+ and CD8+ T cells were then co-cultured with BNL-MEA cells and stimulated with Dynabeads ${ }^{\circledR}$ CD3/CD28 magnetic beads. T-cell proliferation was determined by CFSE dilution measured by flow cytometry (LSRFortessa Flow Cytometer; BD Biosciences) after 3 and 6 days. 


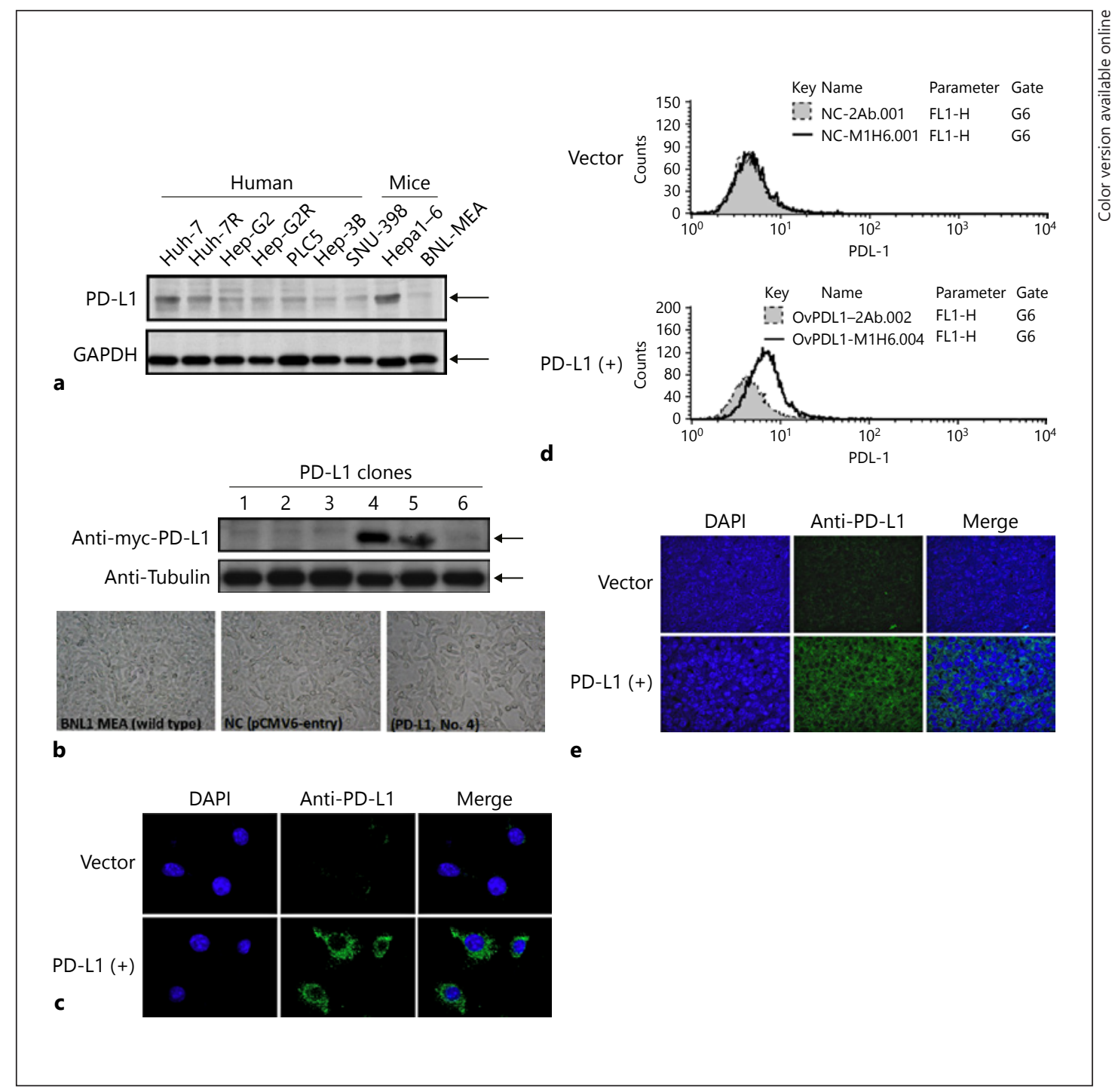

Fig. 1. Expression of PD-L1 in mouse BNL-MEA cells. a PD-L1 expression in wild-type human and mouse HCC cell lines. b BNL-MEA cells were transfected with PD-L1 plasmids (MR203953; Origene Technologies, Rockville, MD, USA), and stable subclones 4 and 5 expressed higher levels of PD-L1 in western blot analysis. c PDL1 was stained by immunofluorescence, and confocal microscopy revealed a greater increase in PD-L1 expression in PD-L1(+) (subclone 4) than in vector. d Flow cytometry showed slightly increased PD-L1 expression on the cell membrane of PD-L1(+) subclone cells (subclone 4). e PD-L1 expression on the PD-L1(+) subclone cells (subclone 4) was confirmed by confocal microscopy.

Fig. 2. PD-L1 expression did not alter in vitro growth characteristics of mouse BNL-MEA cells. a-c PD-L1 expression did not impair cell viability and proliferation characteristics significantly between the stable vector and PD-L1(+) (subclone 4) BNL-MEA cells, as measured by MTT assay (a), colony formation assay (b), and 3D proliferation assay (c). Points represent mean averages $(n=6)$; bars indicate SD. d PD-L1 expression did not alter the proportions in different stages of cell cycle. 


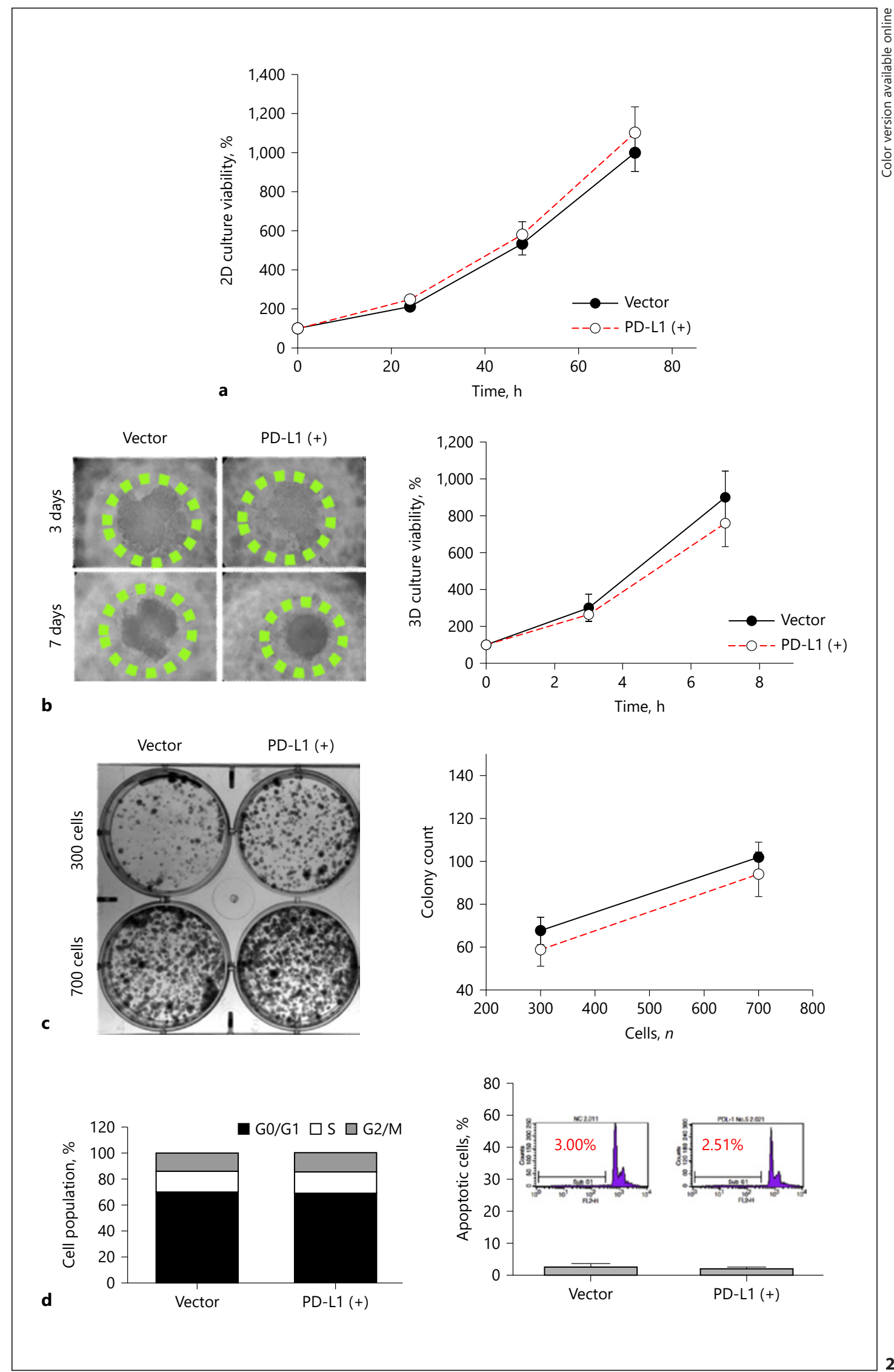

KARGER 


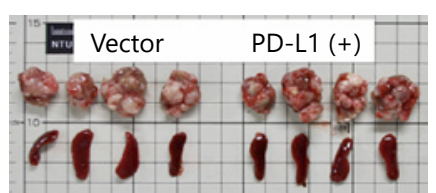

a
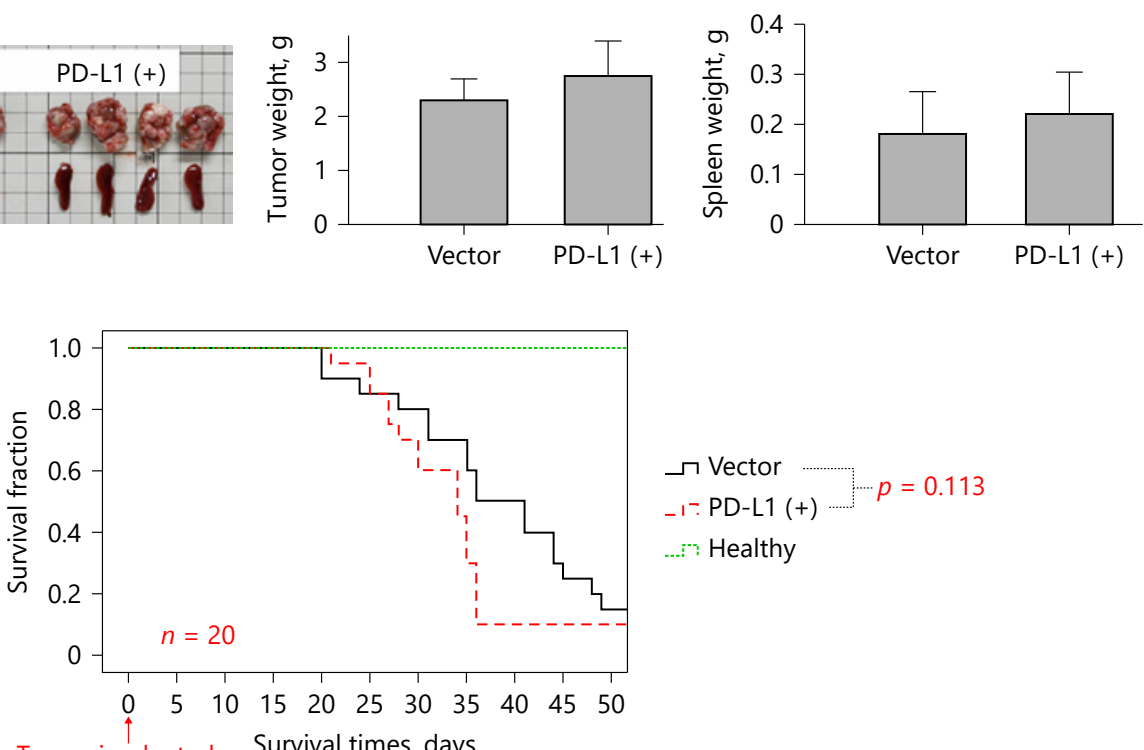

...." Healthy

b

Tumor implanted Survival times, days

Day 10

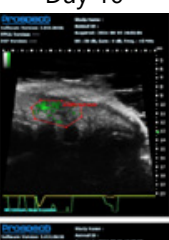

PD-L1 (+)

C

Day 17

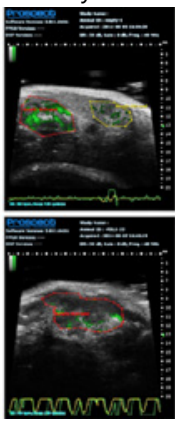

Day 10

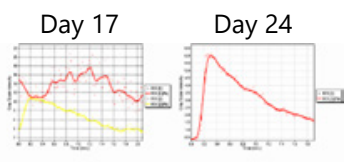

Vector

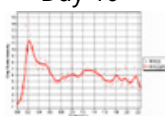

PD-L1 (+)

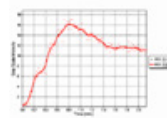

d
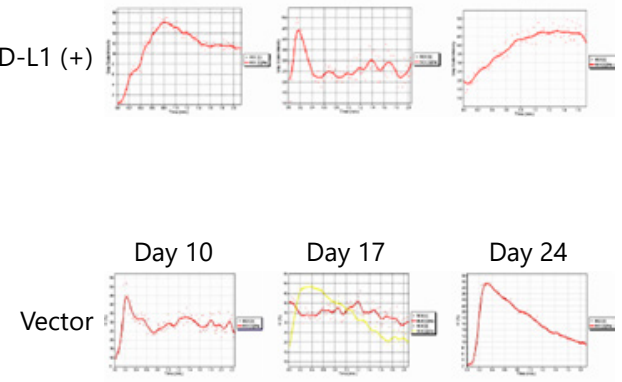

PD-L1 (+)
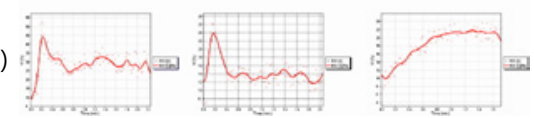

Day 24
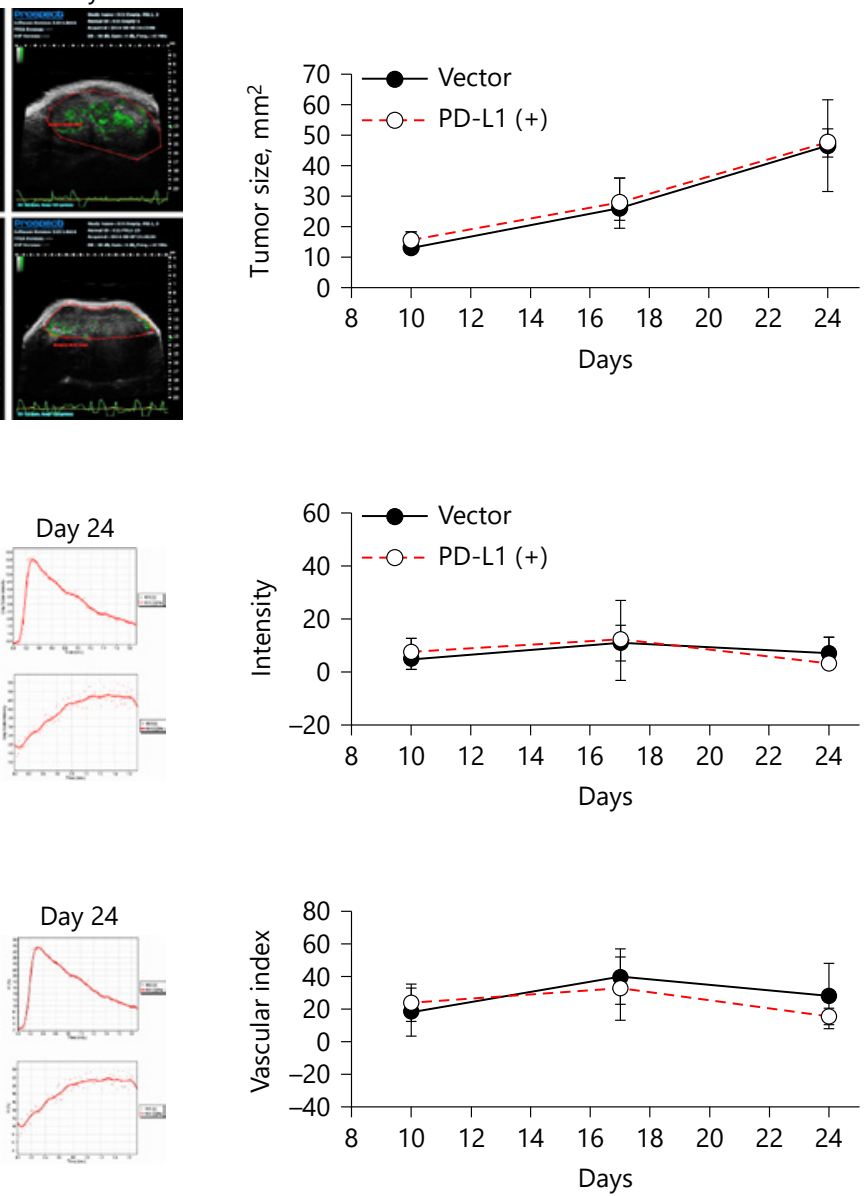


\section{Liver Cancer}

\begin{tabular}{l|l}
\hline Liver Cancer 2019;8:155-171 \\
\hline DOI: 10.1159/000489318 & $\begin{array}{l}\text { @) 2018 S. Karger AG, Basel } \\
\text { www.karger.com/lic }\end{array}$ \\
\hline
\end{tabular}

Ou et al.: PD-L1 Expression in Liver Cancer

\section{Development of Orthotopic Liver Cancer Models}

Orthotopic liver cancer models were established by injecting about $2 \times 10^{5}$ BNL-1MEA cells into the subcapsular area of the left liver lobe of male BALB/c mice at the age of 6-7 weeks, as previously described [26]. The protocol for the animal experiments in this study was approved by the Institutional Animal Care and Use Committee of the College of Medicine, National Taiwan University, and conformed to the criteria outlined in the Guide for the Care and Use of Laboratory Animals, which was prepared by the National Academy of Sciences and published by the National Institutes of Health.

The in vivo growth characteristics of parental and PD-L1-expressing BNL-MEA tumors were compared by using microbubble-enhanced ultrasound (Prospect imaging system; S-Sharp Corporation, Taiwan) [27] and by measuring animal survival. Tumor-bearing mice received intravenous microbubble injection (USphere ${ }^{\mathrm{TM}}$, Trust Bio-sonics, Inc., Hsinchiu, Taiwan) on days 10, 17, and 24 after tumor implantation. With a low-time filtering algorithm, the microbubbles targeted on tumors could be directly visualized, and the arterial vascular signals of the tumors, measured by the time-intensity curve and the vascular index curve, were recorded to estimate tumor perfusion. The expression of immune-related genes in the tumor microenvironment was analyzed by the nCounter PanCancer Immune Profiling Panel (NanoString Technologies, Seattle, WA, USA) [28, 29]. About $50 \mathrm{ng}$ of total RNA from the parental and PD-L1-expressing tumors was used to measure the expression of 730 immune-related genes and 40 housekeeping genes and the results were normalized, by the trimmed mean of M-values normalization method, to estimate the changes of immunerelated genes caused by PD-L1 expression [30]. The changes of representative genes related to T-cell activation and function were confirmed by quantitative reverse transcriptase polymerase chain reaction (qRTPCR) (The primer list for qRT-PCR and detailed methods are described in online suppl. Table S1) [31].

\section{Drug Treatment and Efficacy/Safety Evaluation}

Mice were randomized to different treatment groups on day 5 after tumor implantation. Treatment with anti-mouse PD1 antibody (clone RMP1-14), or isotype control antibody (clone 2A3) (Bio X Cell, West Lebanon, $\mathrm{NH}$, USA), $0.2 \mathrm{mg}$ per dose, was given on days 5, 6, 9, 12, and 15 after tumor implantation by intraperitoneal injection. Sorafenib ( $5 \mathrm{mg} / \mathrm{kg} /$ day; Bayer AG, Leverkusen, Germany) was given daily by gavage. Tumor weights were measured after 21 days of treatment. At the end of treatment, blood samples were collected to measure hemogram and biochemistry, and formalin-fixed, paraffin-embedded tumor samples were collected for analysis of tumor apoptosis (transferase deoxytidyl uridine end labeling [TUNEL] assay), microvessel density, and tumor proliferation (immunohistochemical staining) [24]. To evaluate the roles of CD8+ T cells in the antitumor efficacy of anti-PD1 antibodies and sorafenib, CD8+ T cells were depleted by intraperitoneal injection of anti-CD8 antibody (clone 53-6.72) or isotype control antibody (clone 2A3 or HRPN) (Bio X Cell), respectively. The dosage of antibody treatment was $0.5 \mathrm{mg}$ on day 5 , and then $0.25 \mathrm{mg}$ on days $3,5,7,9,12$, and 15 after tumor implantation. Depletion of CD8+ cells was confirmed by flow cytometry as previously described [26].

\section{Statistical Analysis}

The results of the experiments were expressed as means \pm SD and one-way ANOVA was used to evaluate the statistical significance of the difference between study groups. Animal survival was calculated by the Kaplan-Meier method and compared by log-rank test. Gene set enrichment analysis (GSEA) was performed as previously described [32]. The signal-to-noise ratio metric was used to score the gene expression difference between conditions and the phenotype-based permutation with 1,000 times to obtain $p$ values. The gene sets for GSEA were curated from ConsensusPathDB [33].

Fig. 3. Growth patterns of orthotopic liver tumors were not changed by PD-L1 expression. a BNL-MEA cells with and without PD-L1 expression $\left(2 \times 10^{5}\right.$ cells) were injected into the subcapsular area of the left liver lobe of male BALB/c mice. Tumors were resected after 21 days of injection, and size and volume were measured. $\mathbf{b}$ The median survival time was 36 days in the vector group, 34 days in the PD-L1 (+) group, and >50 days in the healthy group ( $p=0.113$, log-rank test, $n=20$ mice/group). c-e Ultrasound examination of mouse HCC tumor model showed similar trends of tumor size (c), blood perfusion (d), and vascularization index (e) ( $n=5$ in each group). Ultrasound images were facilitated by microbubbles conjugated with antibodies targeting VEGFR (vascular endothelial growth factor receptor). The microbubble targeted on the tumor could be directly visualized (green area) by using a slow-time filtering algorithm. Values are presented as means \pm SD ( $n=5$ in each group). ${ }^{*} p<0.05$, statistically significant (one-way ANOVA). 


\section{Results}

PD-L1 Expression Does Not Alter in vitro and in vivo Growth Characteristics of Liver Cancer Cells

Expression of PD-L1 in human and murine HCC cell lines was examined by western blotting and the BNL-MEA cell line was selected for further experiments because of low endogenous PD-L1 expression (Fig. 1a). PD-L1 expression in the stable clones transfected

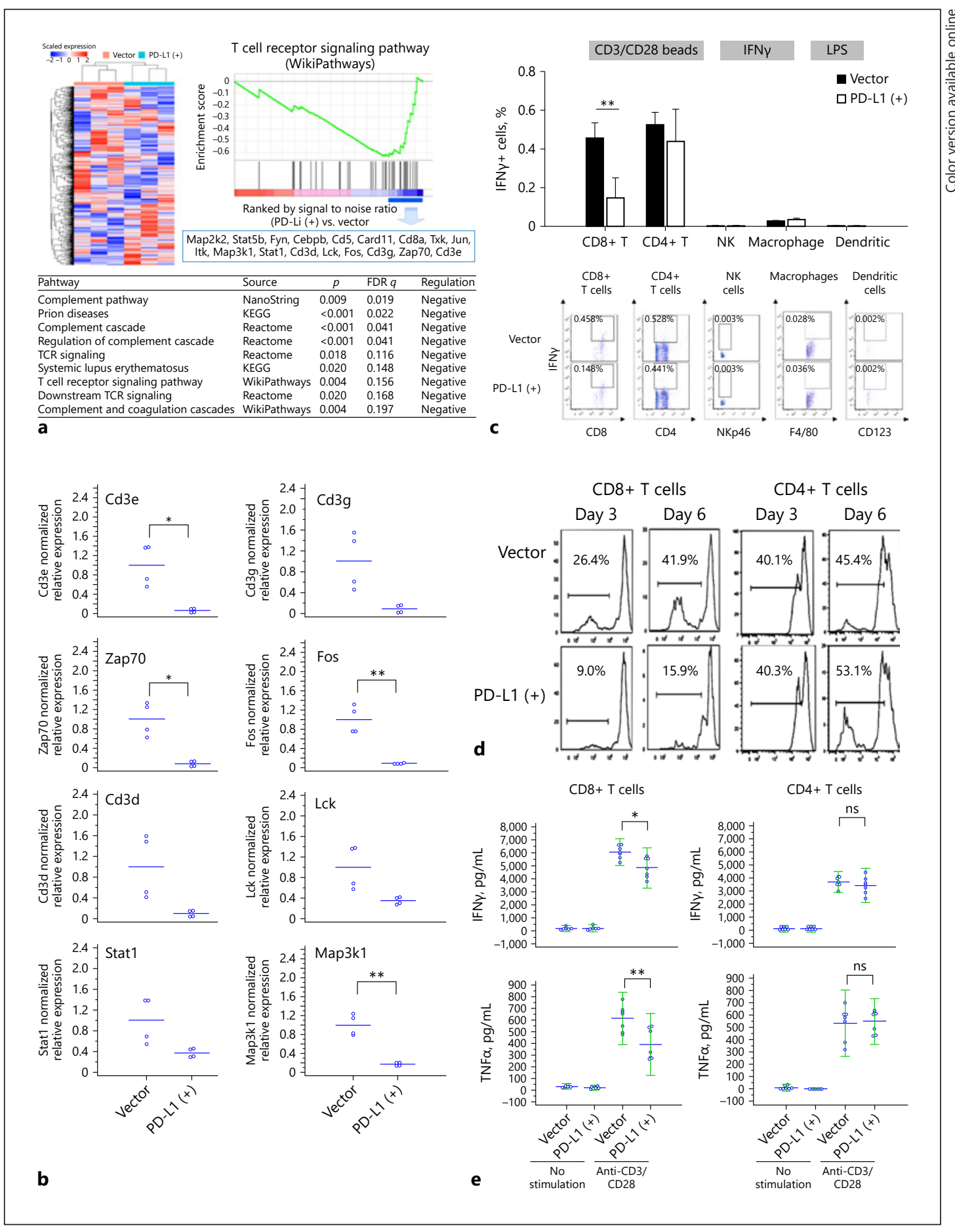

(For legend see next page.) 


\section{Liver Cancer}

\begin{tabular}{l|l}
\hline Liver Cancer 2019;8:155-171 \\
\hline DOI: 10.1159/000489318 & $\begin{array}{l}\text { @) 2018 S. Karger AG, Basel } \\
\text { www.karger.com/lic }\end{array}$ \\
\hline
\end{tabular}

Ou et al.: PD-L1 Expression in Liver Cancer

with PD-L1 plasmids was confirmed by western blotting, confocal immunofluorescence microscopy, and flow cytometry (Fig. 1b-d). Subclone 4 was chosen for further experiments.

The in vitro growth characteristics were similar between the PD-L1-expressing and the parental BNL-MEA cells, as demonstrated by cell viability (Fig. 2a), spheroid formation (Fig. 2b), and colony formation (Fig. 2c). The cell proportions in different stages of cell cycle were also nearly identical (Fig. 2d). The above results indicated that PD-L1 expression in our model did not alter the growth characteristics of liver cancer cells in vitro. Consistent with the in vitro results, there were no significant differences in changes of tumor size, blood perfusion, and animal survival between mice bearing the parental or the PD-L1-expressing liver tumors (Fig. 3), suggesting that expression of PD-L1 did not change the growth pattern of tumors in vivo.

\section{PD-L1 Expression May Suppress Antitumor Immunity in HCC Microenvironment}

The expression of immune-related genes was compared between the parental and PD-L1-expressing mouse liver tumors using the NanoString immune panel to further assess the effects of PD-L1 expression on the liver tumor microenvironment. GSEA indicated that PD-L1 expression significantly downregulated the expression of genes in the T-cell receptor signaling pathway (Fig. 4a). Specifically, genes related to CD8+ T-cell signaling, such as Cd3e, Cd3g, Zap70, Fos, Cd3d, Lck, stat1, and Map3k1, were significantly downregulated, as confirmed by qRT-PCR (Fig. 4b). Results from in vitro co-culture experiments indicated that the proliferation and cytokine secretion (IFN $\gamma$ and TNF $\alpha$ ) of CD8+ T cells were significantly suppressed when co-cultured with PD-L1-expressing BNL-MEA cancer cells, compared with CD8+ T cells co-cultured with parental BNL-MEA cells. On the other hand, the proliferation or cytokine expression of other immune cells, including CD4+ T cells, NK cells, macrophages, or dendritic cells, were not affected significantly (Fig. 4c, d; online suppl. Fig. S1). The results suggested that CD8+ T cells may play critical roles in mediating the immune modulatory effects of PD-L1 expression in the liver microenvironment.

\section{PD-L1-Expressing Liver Tumors May Benefit More from Anti-PD1 Therapy}

The efficacy of anti-PD1 therapy was compared between the parental and PD-L1-expressing orthotopic liver tumors. After anti-PD1 treatment, tumor size and weight reduced more prominently in the PD-L1-expressing tumors than the parental tumors (Fig. 5a). The

Fig. 4. Immune microenvironment in HCC of the orthotopic mouse model. a Genes related to immune cells in PD-L1-expressing and parental tumors were analyzed by gene ontology enrichment analysis and NanoString nCounter ${ }^{\circledR}$ PanCancer Immune Profiling Panel. The heatmap and dendrogram showing distinct gene patterns were generated using GAP software with Pearson correlation and complete linkage. b QRTPCR revealed downregulation of genes related to CD8+ T-cell signaling. Downregulated or upregulated genes were selected under the criteria (1) log fold change $\leq 0.67$ (fold change $<0.2$ ) or $>0.7$ (fold change $>5$ ), (2) $p$ value $<0.1$. c Mouse splenocytes were co-cultured with the BNA-MEA liver cancer cells (with or without PD-L1 expression). The expression of interferon- $\gamma$ (IFN $\gamma$ ) in T cells, NK cells, and macrophage/dendritic cells was measured by intracellular staining and flow cytometry after the splenocytes were stimulated with CD3/ CD28 beads (for T cells), IFN $\gamma$ (for NK cells), or lipopolysaccharides (for macrophage/dendritic cells). IFN $\gamma$ expression in CD8+ T cells was significantly suppressed by PD-L1 expression in liver cancer cells, while those in CD4+ T cells, NK cells, and macrophage/dendritic cells were not affected significantly. d, e CD8+ or CD4+ $\mathrm{T}$ cells isolated from mouse splenocytes were co-cultured with BNL-MEA liver cancer cells with or without PD-L1 expression. In CD8+ T cells proliferation (d) and release of IFN $\gamma$ or tumor necrosis factor- $\alpha(\mathrm{TNF} \alpha)(\mathbf{e})$ after stimulation were significantly suppressed when co-cultured with tumor cells expressing PD-L1. By contrast, in CD4+ T cells neither proliferation nor cytokine release were affected significantly. Values are presented as means \pm SD ( $n=5$ in each group). Statistical significance was set at $* p<0.05, * * p<0.01$, statistically significant (one-way ANOVA). 


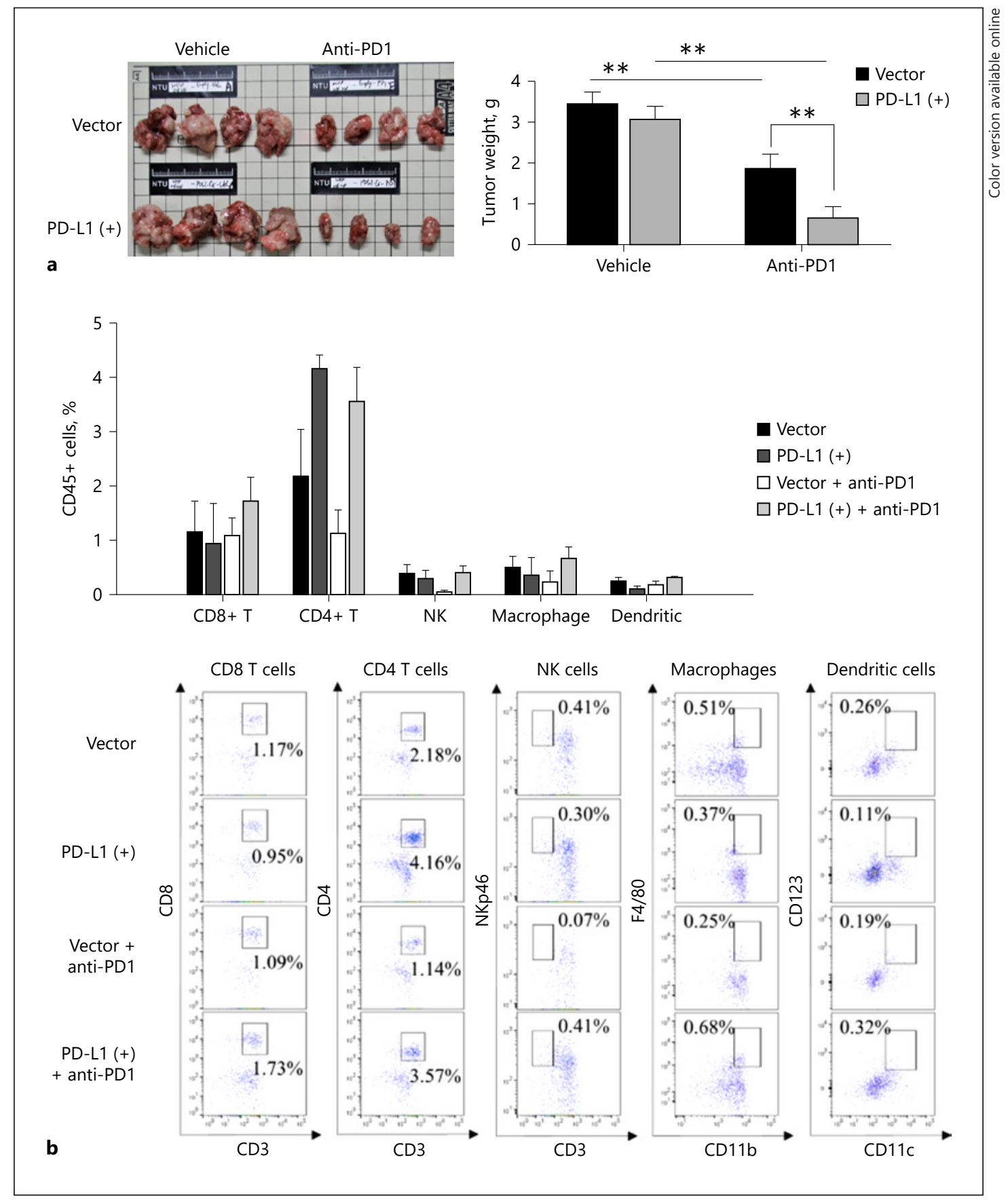

Fig. 5. PD-L1-expressing liver tumors may benefit more from anti-PD1 therapy. a Effects of anti-PD1 therapy on tumor weight. $\mathbf{b}$ Analysis of tumor-infiltrating immune cells after anti-PD1 therapy. Orthotopic liver tumor samples were collected 5 days after treatment start and the percentage of individual immune cell types was measured by flow cytometry. c Analysis of PD1 expression of tumor-infiltrating immune cells by flow cytometry. d Quantification of tumor cell apoptosis (TUNEL assay), microvessel density (MVD), proliferation (Ki67 staining), and CD8+/CD4+ T-cell infiltration by immunohistochemistry using orthotopic liver tumor samples collected 21 days after treatment start. Values are presented as means \pm SD $(n=5$ in each group). was set at ${ }^{* *} p<0.01$, statistically significant (one-way ANOVA).

(Figure continued on next pages.) 


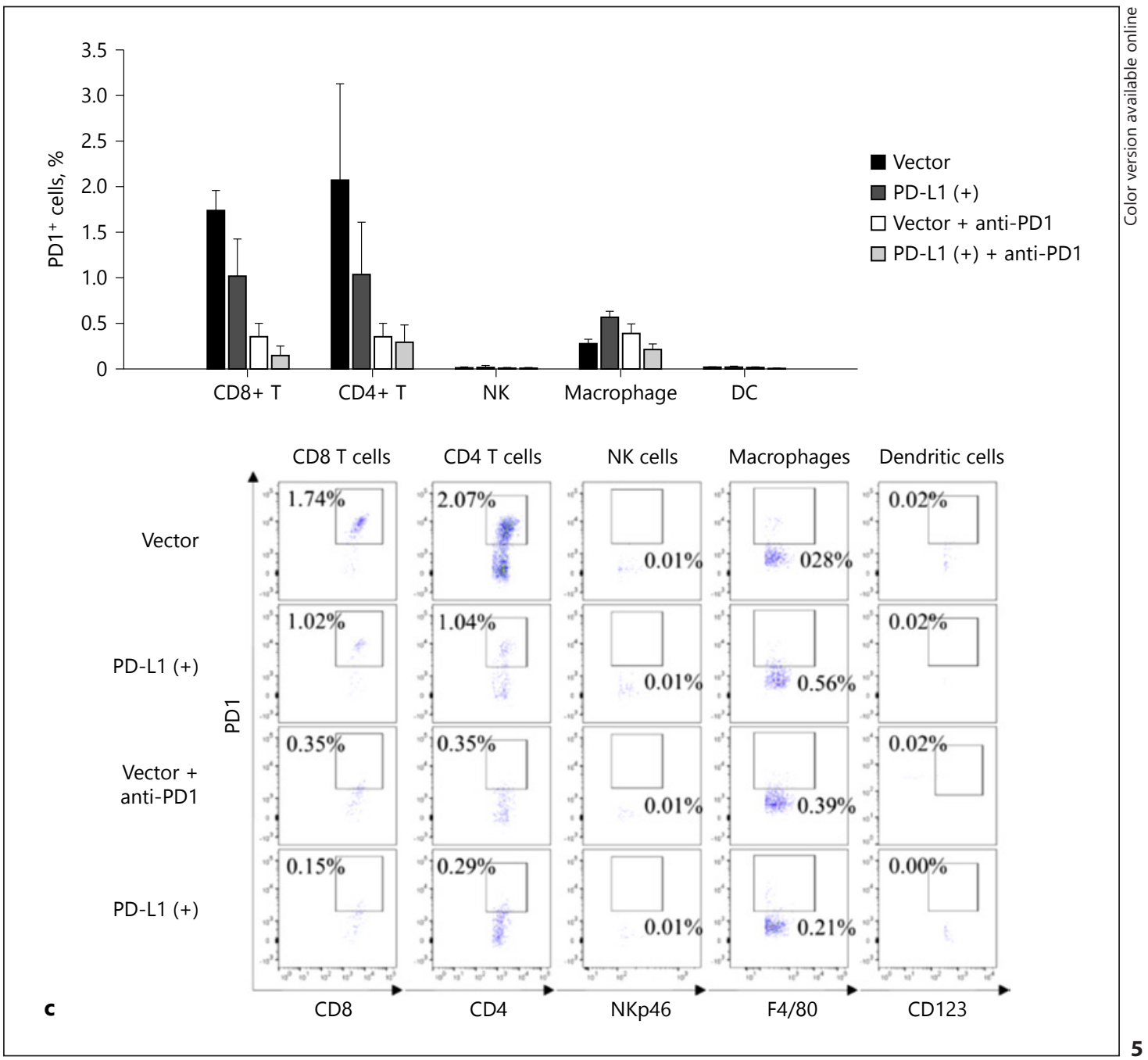

changes in animal body weight, hemogram, or blood chemistry values were similar between mice bearing PD-L1-expressing or parental tumors after anti-PD1 treatment (online suppl. Fig. S2).

The patterns of tumor infiltrating immune cells after anti-PD1 therapy were first analyzed by flow cytometry using the orthotopic liver tumor samples collected 5 days after the start of anti-PD1 treatment. A trend of increase in CD8+ T cells after anti-PD1 therapy was noted in tumors expressing PD-L1. PD-L1 expression in tumor cells increased the numbers of CD4+ T cells, but the effect of anti-PD1 therapy was not obvious (Fig. 5b). Further analysis indicated that the percentage of CD8+ and CD4+ T cells that expressed PD1, a marker associated with the exhaustion phenotype, decreased significantly after anti-PD1 treatment (Fig. 5c). The numbers of NK cells, macrophages, and dendritic cells were low and tumor PD-L1 expression or anti-PD1 therapy did not affect the cell numbers significantly (Fig. 5b, c).

Immunohistochemistry studies were then performed using the orthotopic liver tumor samples collected 21 days after the start of anti-PD1 treatment. PD-L1-expressing tumors may recruit more CD8+ T cells than the parental tumors. The number of both CD8+ and CD4+ $\mathrm{T}$ cells increased after anti-PD1 treatment more prominently in PD-L1-expressing than in parental tumors (Fig. 5d). Tumor PD-L1 expression or anti-PD1 therapy did not affect the 


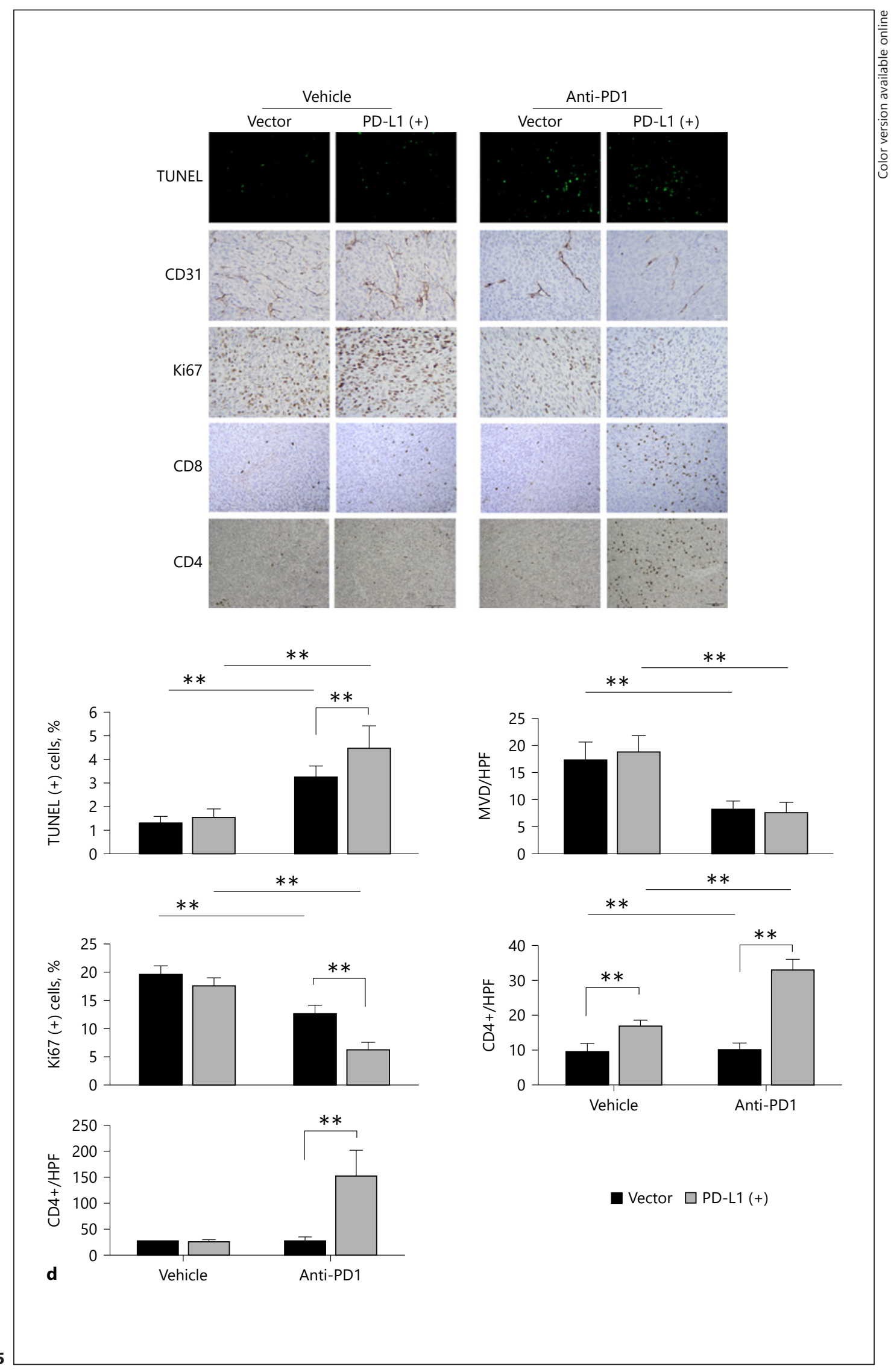

KARGER 


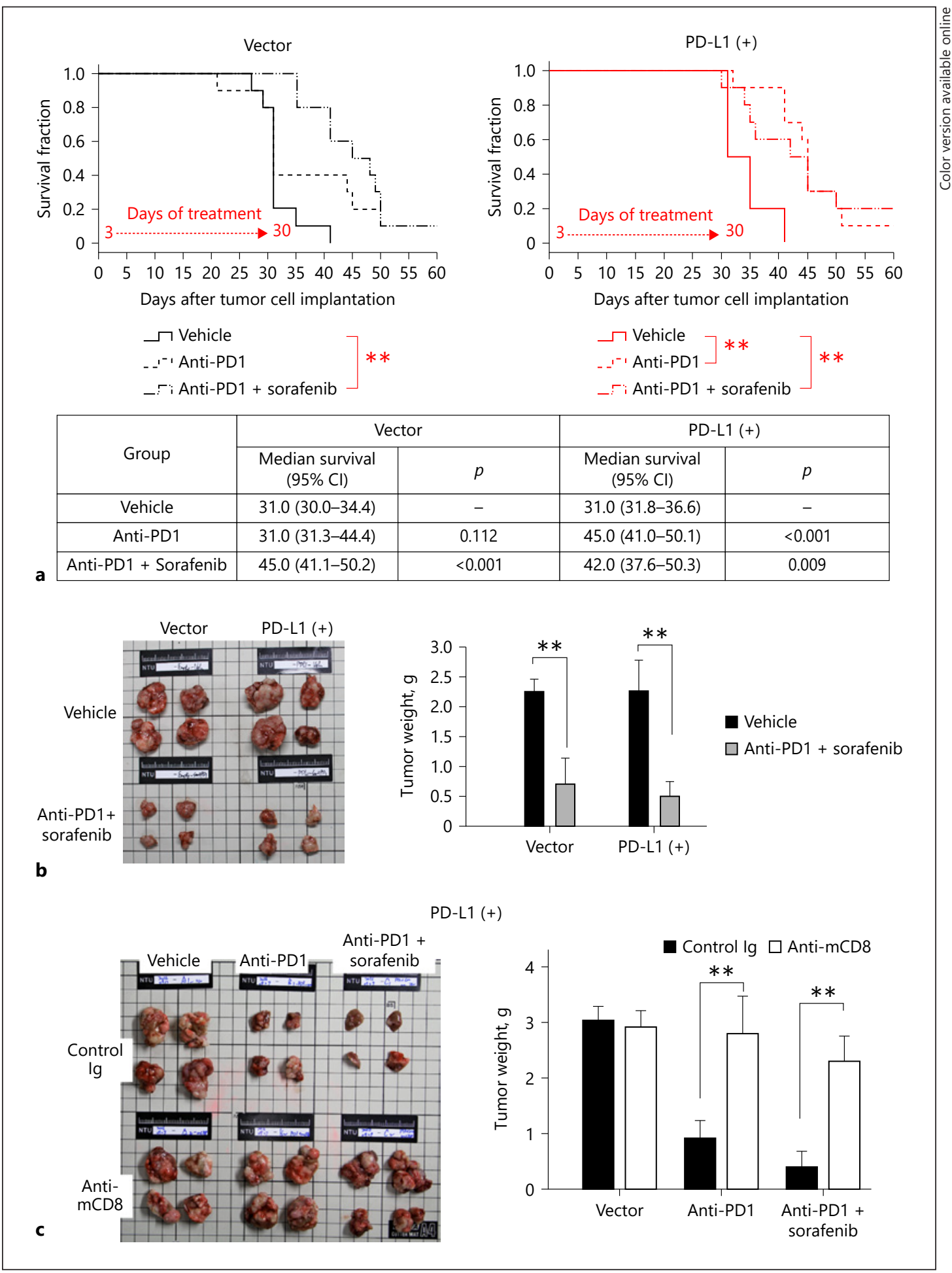

Fig. 6. Antitumor efficacy of anti-PD1 plus sorafenib treatment. a Anti-PD1 therapy significantly improved survival only in mice with PD-L1-expressing tumors, while combination therapy was effective against both parental (vector) and PD-L1-expressing tumors. ${ }^{* *} p<0.01$. b Combination of anti-PD1 therapy and sorafenib could reduce tumor size and weight in both PD-L1-expressing and parental tumors ( $n=4$ in each group). c The weight of PD-L1-expressing tumors treated with anti-PD1 therapy was significantly increased when the tumor was depleted of CD8+ T cells with anti-mouse CD8+ T cell antibodies ( $n=5$ in each group). Values are presented as means \pm SD. was set at ${ }^{* *} p<0.01$, statistically significant (one-way ANOVA). 


\section{Liver Cancer}

numbers of NK cells, macrophages, and dendritic cells significantly (online suppl. Fig. S3). The inconsistent findings in the patterns of T-cell changes between flow cytometry and immunohistochemistry experiments may result partly from the different time points chosen for tumor sampling. Anti-PD1 treatment significantly increased tumor cell apoptosis and decreased tumor cell proliferation, both of which were more prominent in PD-L1-expressing tumors, but the extent of decrease in tumor microvessel density was similar between mice bearing PD-L1-expressing or parental tumors (Fig. 5d). The results suggested that PD-L1 expression may affect the efficacy of anti-PD1 therapy for HCC and the ability to activate T cells in the tumor microenvironment.

The mice bearing PD-L1-expressing liver tumors showed significantly better survival time after anti-PD1 treatment, while mice bearing parental tumors showed similar survival times between those treated with anti-PD1 or vehicle (Fig. 6a). When anti-PD1 was combined with sorafenib, the current standard targeted therapy for advanced HCC, the mice bearing either parental or PD-L1-expressing tumors showed a similar extent of tumor regression and improvement in survival (Fig. 6a, b). Mice treated with the combination regimen showed similar safety profiles in terms of body weight, hemogram, and blood chemistry data (see online suppl. Fig. S4). The data suggested that resistance caused by PD-L1 expression in HCC can be overcome by combination with sorafenib. The role of CD8+ T cells in antitumor activity was further confirmed by the depletion experiment using anti-CD8 antibodies. The efficacy of depletion of CD8+ T cells was demonstrated by flow cytometry (see online suppl. Fig. S5). The antitumor efficacy of anti-PD1 treatment, alone or in combination with sorafenib, was abolished by depletion of CD8+ T cells (Fig. 6c).

\section{Discussion}

In this study, the impact of PD-L1 expression in HCC cells on the liver tumor microenvironment was explored. PD-L1 expression in our model did not affect the growth characteristics of HCC cells in vitro or in vivo. Although PD-L1 expression was associated with increased numbers of CD8+ T cells in the liver tumor microenvironment, their functions in terms of cell proliferation and cytokine secretion were inhibited. Increased PD-L1 expression was associated with better efficacy of anti-PD1 therapy. Our model also supports the potential of combination of anti-PD1 therapy and targeted therapy for HCC.

Effects of PD-L1 expression in the tumor microenvironment have been investigated in multiple preclinical models, in which PD-L1 expression was induced by either genetic aberration [19] or activation of upstream oncogenic signaling pathways. PD-L1 expression and activation of PD1/PD-L1 signaling can enhance immune evasion by inhibition of recruitment and activation of $\mathrm{T}$ cells and macrophages, thus further promoting tumor growth. Our model demonstrated that constitutive PD-L1 expression on liver cancer cells may reduce cytotoxic T-cell activation and function in the liver tumor microenvironment, suggesting that our model may be useful in future mechanistic exploration of anti-PD1-based therapy for HCC. The association between PD-L1 expression and the efficacy of anti-PD1 therapy in our model supports the findings from the nivolumab trial, in which the objective response rate was, albeit statistically insignificant, numerically higher for patients with PD-L1 (+) tumors (27 vs. $12 \%$ in the escalation cohort and 26 vs. $19 \%$ in the expansion cohort) $[8,9]$. However, the predictive value of PD-L1 expression for the efficacy of anti-PD1/anti-PD-L1 therapy may be confounded by many technical issues, including differences in antibodies and staining protocol used, criteria (cutoff value) of positivity, target cells (cancer cells vs. immune cells) for evaluation, and interobserver reproducibility [34]. Further investigation of the full biological impact of PD-L1 expression in HCC is warranted. 


\section{Liver
Cancer}

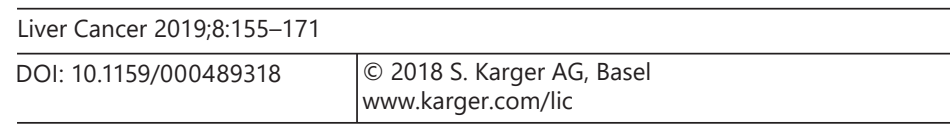

Ou et al.: PD-L1 Expression in Liver Cancer

A potential use of our model is to test the antitumor synergy between anti-PD1 and other systemic therapies. Our data suggested that the combination of anti-PD1 and sorafenib may have antitumor synergy, irrespective of the PD-L1 expression status in the tumor cells. In our model anti-PD1 therapy could reduce PD1+ T cells in the tumor microenvironment irrespective of PD-L1 expression, suggesting that direct T-cell activation may account for the efficacy of anti-PD1 therapy, even in tumors without PD-L1 expression. T-cell activation by checkpoint inhibitor therapy may directly improve vascular normalization in tumors and may thus enhance the response to antiangiogenic therapy [35, 36]. Combining sorafenib or other antiangiogenic agents with checkpoint inhibitor therapy may increase the influx of CD8+ T cells over Treg cells and attenuate tumor-induced immunosuppression via reversal of tumor angiogenesis [37, 38], and an objective response rate of more than $50 \%$ has been seen in patients with metastatic renal cell carcinoma $[39,40]$. No evident increase in toxicity with the combination therapy was noted in our model. However, data from early-phase clinical trials indicated that a combination of checkpoint inhibitor therapy and molecular targeted therapy may augment the toxicity of individual agents $[41,42]$. The safety issues must be carefully clarified in further clinical trials.

The potential antitumor synergy between checkpoint inhibitor treatment and targeted therapy may also be affected by the specific model microenvironment. Chen et al. [43] reported no antitumor synergy between anti-PD1 and sorafenib in their orthotopic HCC model, which used $\mathrm{CCl} 4$ gavage to induce liver fibrosis before orthotopic implantation of the HCA- 1 cells [43]. This model was characterized by SDF- $1 \alpha / C-X-C$ receptor type 4 (CXCR4)dependent infiltration of myeloid cells in the tumor microenvironment, which may offset the potential antitumor synergy between anti-PD1 and sorafenib or other targeted agents. A recent transcriptomic study of the immune characteristics of HCC tumors and the surrounding liver indicated that the immune contexture of the surrounding liver was mostly immunosuppressive and associated with inferior survival of HCC patients [44]. Prospective biomarker studies within the context of drug clinical trials are necessary to clarify whether the inflammatory microenvironment in HCC patients with underlying cirrhosis may negate the immunomodulatory effects of antiangiogenic agents.

There are several limitations to our model. Firstly, PD-L1 may be overexpressed in not only HCC tumor cells but also inflammatory cells in the tumor microenvironment, and PD-L1 expression in either tumor cells or inflammatory cells were associated with aggressive tumor behavior [18]. Secondly, the chronic inflammation associated with different etiologies (viral hepatitis, alcohol, nonalcoholic steatohepatitis, etc.) may impact on different immune cells and cytokine network in the liver microenvironment [45], which is difficult to reproduce in preclinical models. Thirdly, while PD-L1 expression in our model may suppress cytotoxic T-cell function, we found an apparently paradoxical increase in the numbers of tumor-infiltrating CD8+ T cells. Recruitment of effector T cells is regulated by a complex interaction among the expression of various checkpoint proteins, the cytokine network, as well as dendritic cells and other cellular components in the tumor microenvironment [46-48]. Because of a paucity of relevant preclinical models, this type of study is just emerging for HCC. A more integrative analysis of intratumoral cell distribution is required to characterize the interaction between PD-L1 expression, immune cell infiltration, and responses to anti-PD1 therapy in HCC.

In conclusion, the PD-L1-expressing mouse HCC model we generated may be used to investigate how PD-L1 may modulate the immune contexture of HCC. Anti-PD1 therapy appeared more effective in PD-L1-expressing than nonexpressing tumors, but the difference was diminished by the addition of sorafenib. 


\section{Liver Cancer}

\section{Acknowledgments}

The authors thank the Laboratory Animal Center, College of Medicine, National Taiwan University, for support with the animal studies. They also thank Dr. Ping-Chih Ho, Department of Fundamental Oncology, University of Lausanne, Switzerland, for his intellectual input into the study design and provision of experimental material. The authors are grateful to the Liver Disease Prevention \& Treatment Foundation, Taiwan, for logistic support, the Second Core Laboratory, Department of Medical Research, National Taiwan University Hospital for technical assistance with the confocal microscopic images, and the First Core Laboratory, National Taiwan University College of Medicine for technical assistance with the flow cytometry. This study was supported by grants from the Ministry of Science and Technology, Taiwan (MOST 103-2314-B-002112-MY3, MOST 105-2314-B-002-189-MY3, MOST 105-2314-B-002-199, and MOST 106-2314-B-002229-MY3), and from the National Taiwan University (YongLin Chair Grant S-01).

\section{Disclosure Statement}

Dr. Ann-Lii Cheng is a consultant for and a member of the speaker's bureau of Bayer-Schering Pharma. He is also a consultant of Novartis, Merck Serono, Eisai, Merck Sharp \& Dohme (I.A.) Corp., ONXEO, Bayer HealthCare Pharmaceuticals Inc., Bristol-Myers Squibb Company, and Ono Pharmaceutical Co., Ltd. Dr. Chiun Hsu has received a research grant from Celgene and has received honorarium from the following pharmaceutical companies: AstraZeneca, Bayer, Bristol-Myers Squibb/ONO, Eli Lilly, MSD, Novartis, Roche, and TTY Biopharm. All other authors have no conflicts of interest relevant to this manuscript to disclose.

\section{References}

1 Topalian SL, Drake CG, Pardoll DM: Immune checkpoint blockade: a common denominator approach to cancer therapy. Cancer Cell 2015;27:450-461.

2 Callahan MK, Postow MA, Wolchok JD: Targeting T cell co-receptors for cancer therapy. Immunity 2016;44: 1069-1078.

3 Khalil DN, et al: The future of cancer treatment: immunomodulation, CARs and combination immunotherapy. Nat Rev Clin Oncol 2016;13:273-290.

4 Forner A, Llovet JM, Bruix J: Hepatocellular carcinoma. Lancet 2012;379:1245-1255.

5 Bruix J, et al: Regorafenib for patients with hepatocellular carcinoma who progressed on sorafenib treatment (RESORCE): a randomised, double-blind, placebo-controlled, phase 3 trial. Lancet 2017;389:56-66.

6 Prieto J, Melero I, Sangro B: Immunological landscape and immunotherapy of hepatocellular carcinoma. Nat Rev Gastroenterol Hepatol 2015;12:681-700.

7 Sangro B, et al: A clinical trial of CTLA-4 blockade with tremelimumab in patients with hepatocellular carcinoma and chronic hepatitis C. J Hepatol 2013;59:81-88.

8 Crocenzi TS, El-Khoueiry AB, Yau T, et al: Nivolumab in Sorafenib-Naive and -Experienced Patients With Advanced Hepatocellular Carcinoma: CheckMate 040 Study. 2017 ASCO Annual Meeting. Chicago, June 2-6, 2017, Abstract 4013.

9 El-Khoueiry AB, et al: Nivolumab in patients with advanced hepatocellular carcinoma (CheckMate 040): an open-label, non-comparative, phase 1/2 dose escalation and expansion trial. Lancet 2017;389:2492-2502.

10 Teng MW, et al: Classifying cancers based on T-cell infiltration and PD-L1. Cancer Res 2015;75:2139-2145.

11 Ribas A, Hu-Lieskovan S: What does PD-L1 positive or negative mean? J Exp Med 2016;213:2835-2840.

12 Patel SP, Kurzrock R: PD-L1 Expression as a predictive biomarker in cancer immunotherapy. Mol Cancer Ther 2015;14:847-856.

13 Herbst RS, etal: Pembrolizumab versus docetaxel for previously treated, PD-L1-positive, advanced non-smallcell lung cancer (KEYNOTE-010): a randomised controlled trial. Lancet 2016;387:1540-1550.

14 Nanda R, etal: Pembrolizumab in patients with advanced triple-negative breast cancer: phase Ib KEYNOTE-012 study. J Clin Oncol 2016;34:2460-2467.

15 Gandini S, Massi D, Mandala M: PD-L1 expression in cancer patients receiving anti PD-1/PD-L1 antibodies: a systematic review and meta-analysis. Crit Rev Oncol Hematol 2016;100:88-98.

16 McLaughlin J, et al: Quantitative assessment of the heterogeneity of PD-L1 expression in non-small-cell lung cancer. JAMA Oncol 2016;2:46-54.

17 Madore J, et al: PD-L1 expression in melanoma shows marked heterogeneity within and between patients: implications for anti-PD-1/PD-L1 clinical trials. Pigment Cell Melanoma Res 2015;28:245-253.

18 Calderaro J, et al: Programmed death ligand 1 expression in hepatocellular carcinoma: relationship with clinical and pathological features. Hepatology 2016;64:2038-2046. 
Ou et al.: PD-L1 Expression in Liver Cancer

19 Kataoka K, et al: Aberrant PD-L1 expression through 3'-UTR disruption in multiple cancers. Nature 2016;534: 402-406.

20 Casey SC, et al: MYC regulates the antitumor immune response through CD47 and PD-L1. Science 2016;352: 227.

21 Dorand RD, et al: Cdk5 disruption attenuates tumor PD-L1 expression and promotes antitumor immunity. Science 2016;353:399.

22 Akbay EA, et al: Activation of the PD-1 pathway contributes to immune escape in EGFR-driven lung tumors. Cancer Discov 2013;3:1355.

23 Lastwika KJ, et al: Control of PD-L1 expression by oncogenic activation of the AKT-mTOR pathway in nonsmall cell lung cancer. Cancer Res 2016;76:227.

24 Ou DL, et al: Potential synergistic anti-tumor activity between lenalidomide and sorafenib in hepatocellular carcinoma. J Gastroenterol Hepatol 2014;29:2021-2031.

25 Lai C-Y, et al: Tyrosine phosphorylation of c-Maf enhances the expression of IL-4 gene. J Immunol 2012;189: 1545-1550.

26 Chang CJ, et al: Combined GM-CSF and IL-12 gene therapy synergistically suppresses the growth of orthotopic liver tumors. Hepatology 2007;45:746-754.

27 Peng HH, et al: Real-time monitoring of inertial cavitation effects of microbubbles by using MRI: in vitro experiments. Magn Reson Med 2017;77:102-111.

28 Prat A, et al: Immune-related gene expression profiling after PD-1 blockade in non-small cell lung carcinoma, head and neck squamous cell carcinoma and melanoma. Cancer Res 2017;77:3540-3550.

29 Geiss GK, et al: Direct multiplexed measurement of gene expression with color-coded probe pairs. Nat Biotechnol 2008;26:317-325.

30 Robinson MD, Oshlack A: A scaling normalization method for differential expression analysis of RNA-seq data. Genome Biol 2010;11:R25.

31 Hsu C, et al: Cyclin E1 inhibition can overcome sorafenib resistance in hepatocellular carcinoma cells through Mcl-1 suppression. Clin Cancer Res 2016;22:2555-2564.

32 Subramanian A, et al: Gene set enrichment analysis: a knowledge-based approach for interpreting genomewide expression profiles. Proc Natl Acad Sci USA 2005;102:15545-15550.

33 Kamburov A, et al: The ConsensusPathDB interaction database: 2013 update. Nucleic Acids Res 2013; 41(Database issue):D793-D800.

34 Buttner R, et al: Programmed death-ligand 1 immunohistochemistry testing: a review of analytical assays and clinical implementation in non-small-cell lung cancer. J Clin Oncol 2017;35:3867-3876.

35 Tian L, et al: Mutual regulation of tumour vessel normalization and immunostimulatory reprogramming. Nature 2017;544:250-254.

36 Khan KA, Kerbel RS: Improving immunotherapy outcomes with anti-angiogenic treatments and vice versa. Nat Rev Clin Oncol 2018;15:310-324.

37 Motz GT, et al: Tumor endothelium FasL establishes a selective immune barrier promoting tolerance in tumors. Nat Med 2014;20:607-615.

38 Chen DS, Mellman I: Oncology meets immunology: the cancer-immunity cycle. Immunity 2013;39:1-10.

39 Choueiri TK, et al: First-line avelumab + axitinib therapy in patients (pts) with advanced renal cell carcinoma (aRCC): results from a phase Ib trial. J Clin Oncol 2017;35(suppl 15):4504-4504.

40 Larkin J, et al: Phase 1b dose-finding study of avelumab (anti-PD-L1) + axitinib in treatment-naïve patients with advanced renal cell carcinoma. Annals of Oncology 2016;27(suppl 6):775PD-775PD.

41 Ribas A, et al: Hepatotoxicity with combination of vemurafenib and ipilimumab. N Engl J Med 2013;368:13651366.

42 Amin A, Plimack ER, Infante J, Ernstoff B, Rini BI: Nivolumab (anti-PD-1; BMS-936558, ONO-4538) in combination with sunitinib or pazopanib in patients (pts) with metastatic renal cell carcinoma (mRCC) (abstract). J Clin Oncol 2014;32(suppl 5s):5010.

43 Chen Y, et al: CXCR4 inhibition in tumor microenvironment facilitates anti-programmed death receptor-1 immunotherapy in sorafenib-treated hepatocellular carcinoma in mice. Hepatology 2015;61:1591-1602.

44 Sia D, et al: Identification of an immune-specific class of hepatocellular carcinoma, based on molecular features. Gastroenterology 2017;153:812-826.

45 Ringelhan M, et al: The immunology of hepatocellular carcinoma. Nat Immunol 2018;19:222-232.

46 Spranger $\mathrm{S}$, et al: Tumor-residing Batf3 dendritic cells are required for effector $\mathrm{T}$ Cell trafficking and adoptive T cell therapy. Cancer Cell 2017;31:711-723.e4.

47 Petit AE, et al: A major secretory defect of tumour-infiltrating T lymphocytes due to galectin impairing LFA1-mediated synapse completion. Nat Commun 2016;7:12242.

48 Chen DS, Mellman I: Elements of cancer immunity and the cancer-immune set point. Nature 2017;541:321330. 